\title{
Bridging to CAR-T Cells in Children, Adolescents, and Young Adults with ALL
}

\author{
André Baruchel
}

\section{The Importance of the Bridge}

Leukapheresis can be performed in most patients, including infants, but not all patients will receive autologous CAR-T cells. The ELIANA trial can be taken as an example to help understand the issues: 97 patients were successfully screened and enrolled, but only 79 of them finally made it to the infusion. Of the remaining 18 patients, 10 died or experienced an AE during the manufacturing time, and 8 patients had issues with the manufacturing process (Grupp et al. 2018).

Contrary to what is expected prior to allogeneic haematopoietic cell transplantation (allo-HCT), the role of an optimal bridging therapy is not to obtain the lowest residual disease but only a reduction or stabilization of tumour burden, bringing the patient to the CAR-T cell infusion in good clinical condition.

Some facts are to recall:

- The interval between apheresis and infusion is highly variable and during clinical trials can range between 3 weeks and 3 months. With the approved commercial product tisagenlecleucel, the interval is now in the 3-4-week range. Of note, manufacturing could be shorter in academic closed systems using fresh cells and decentralized manufacturing.

- Many variables can indeed influence the final interval:

- Cryopreservation to shipping: time can be lost, being influenced by manufacturing slots.

- Manufacturing site: USA vs. EU.

\footnotetext{
A. Baruchel (ه)

Université de Paris et EA 3518 Institut de Recherche Saint-Louis, Paris, France

Paris, France

e-mail: andre.baruchel@rdb.aphp.fr

Service d'Hémato-Immunologie Pédiatrique, Hôpital Universitaire Robert Debré (APHP), 
- Availability of the cell therapy lab to receive the apheresis product and then the common availability of the same lab and the pharmacists to receive the "drug".

- Availability of a room in the clinical department and the possibility that the patient can be admitted to the ICU in the case of complications, which can be an issue in the current pandemic.

- Of utmost importance are the course of the disease and the clinical status, with the main risks being disease progression, occurrence of infection (fungal diseases in particular) in these heavily pretreated patients and other SAEs linked to chemo/immunotherapies (e.g., inotuzumab).

\section{Thus, the « art of bridging » includes the following steps:}

- Selecting the best chemotherapy requires integrating the disease biology, previous disease sensitivity to treatment and the tolerance history of the patient. There is no « one size fits all » here.

- Aiming to undergo lymphodepletion plus infusion without too much disease as a way to decrease the incidence of CRS and increase the final outcome.

- Monitoring a patient who is not completely under your control: close collaboration and numerous contacts (at least 2/week) with the referring centre are mandatory.

\section{Examples of possible choices are found in Table 21.1.}

Table 21.1 Possible bridging therapies on the road to CAR-T cell therapy according to tumour burden and disease localization and kinetics ${ }^{\mathrm{a}}$

No treatment: smouldering disease

Low-intensity chemotherapy: low disease burden and/or slowly progressing ALL

- Weekly vincristine (VCR) with oral 6MP and methotrexate (MTX).

-Weekly VCR plus dexamethasone (DEX) $6 \mathrm{mg} / \mathrm{m}^{2} 2$ days/week.

Intermediate-intensity chemotherapy: disease burden and/or progressing ALL

- Consolidation «IB » (6MP, cytarabine, cyclophosphamide).

-Weekly VCR plus DEX, bortezomib, asparaginase.

High-intensity chemotherapy: aggressive disease or $\mathrm{EMD}^{\mathrm{b}}$

- High-dose (HD) cytarabine, VP16-cyclophosphamide, hyper CVAD.

- High-dose MTX if CNS involvement.

Very high-intensity chemotherapy for rapidly progressing disease:

- Sequential approach, e.g., HD cytarabine followed by LD.

a Targeted agents, such as TKIs, can be used in Ph+ ALL and ABL class fusion ALL in addition to low-intensity chemotherapy, for example

${ }^{\mathrm{b}}$ EMD: extramedullary disease 


\section{Is There a Place for Immunotherapy?}

- Accumulating data suggest that the use of CD19-oriented therapy (e.g., blinatumomab) prior to administration of CD19 CAR-T cells can be detrimental, particularly with the selection of CD19-negative clones (Pillai et al. 2019; Baruchel et al. 2020; Taraseviciute et al. 2020).

- Inotuzumab ozogamicin, an anti-CD22 therapy, can be used in patients with chemoresistant disease but can result in a high rate of negative MRD, sometimes with no remaining normal B cells, which could theoretically lead to an insufficient target level for adequate CAR-T cell expansion and persistence. In a recent clinical trial (Baruchel et al. 2020), this treatment was associated with a diminished EFS. Inotuzumab ozogamicin is also not recommended prior to the use of anti-CD22 CAR-T cells.

\section{Key Points}

- The aim of optimal bridging therapy is not to obtain the lowest residual disease possible but only to reduce or stabilize the tumour burden, bringing the patient to CAR-T cell infusion in good clinical condition.

- There is no "one size fits all" in this area. Deep knowledge of disease biology, emerging targets, previous sensitivity to treatment, and the tolerance history of the patient are needed.

\section{References}

Baruchel A, Krueger J, Balduzzi A. Tisagenlecleucel for pediatric/young adult patients with relapsed/refractory b-cell acute lymphoblastic leukemia: preliminary report of B2001X focusing on prior exposure to blinatumomab and inotuzumab. EHA Library. 2020;294938:S118.

Grupp SA, Maude SL, Rives S, et al. Updated analysis of the efficacy and safety of tisagenlecleucel in pediatric and young adult patients with relapsed/refractory $(\mathrm{r} / \mathrm{r})$ acute lymphoblastic leukemia. Blood. 2018;132(Suppl 1):895.

Pillai V, Muralidharan K, Meng W, et al. CAR-T cell therapy is effective for CD19-dim B-lymphoblastic leukemia but is impacted by prior blinatumomab therapy. Blood Adv. 2019;3:3539-49.

Taraseviciute A, Steinberg SM, Myers RM, et al. Pre-CAR blinatumomab is associated with increased post-CD19 CAR relapse and decreased event free survival. Blood. 2020;136(Suppl 1):13-4. 
Open Access This chapter is licensed under the terms of the Creative Commons Attribution 4.0 International License (http://creativecommons.org/licenses/by/4.0/), which permits use, sharing, adaptation, distribution and reproduction in any medium or format, as long as you give appropriate credit to the original author(s) and the source, provide a link to the Creative Commons license and indicate if changes were made.

The images or other third party material in this chapter are included in the chapter's Creative Commons license, unless indicated otherwise in a credit line to the material. If material is not included in the chapter's Creative Commons license and your intended use is not permitted by statutory regulation or exceeds the permitted use, you will need to obtain permission directly from the copyright holder.

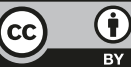

